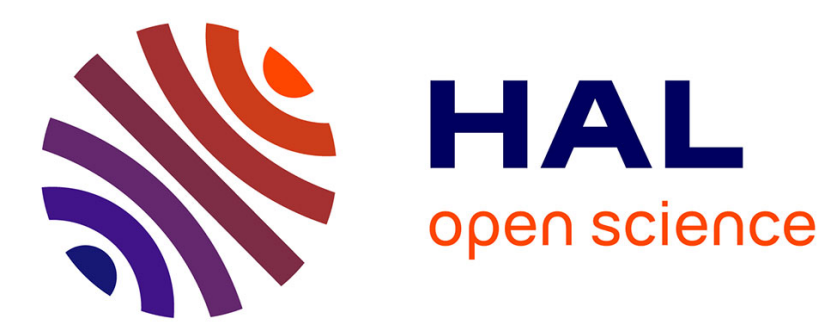

\title{
DC4LED: A Hierarchical VDTN Routing for Data Collection in Smart Cities
}

\author{
Ngurah Indra Er, Kamal Deep Singh, Jean-Marie Bonnin
}

\section{To cite this version:}

Ngurah Indra Er, Kamal Deep Singh, Jean-Marie Bonnin. DC4LED: A Hierarchical VDTN Routing for Data Collection in Smart Cities. CCNC 2019 - 16th IEEE Consumer Communications and Networking Conference, Jan 2019, Las Vegas, United States. pp.883-886, 10.1109/CCNC.2019.8651757 . hal-01894719v2

\section{HAL Id: hal-01894719 https://hal.science/hal-01894719v2}

Submitted on 5 Mar 2019

HAL is a multi-disciplinary open access archive for the deposit and dissemination of scientific research documents, whether they are published or not. The documents may come from teaching and research institutions in France or abroad, or from public or private research centers.
L'archive ouverte pluridisciplinaire HAL, est destinée au dépôt et à la diffusion de documents scientifiques de niveau recherche, publiés ou non, émanant des établissements d'enseignement et de recherche français ou étrangers, des laboratoires publics ou privés. 


\section{DC4LED: A Hierarchical VDTN Routing for Data Collection in Smart Cities}

\author{
Ngurah Indra ER \\ IRISA / IMT-Atlantique, Rennes-France \\ $\&$ \\ Udayana University, Bali-Indonesia \\ indra@unud.ac.id
}

\author{
Kamal Deep SINGH \\ Laboratoire Hubert Curien \\ Universite Jean Monnet \\ Saint-Etienne - France \\ kamal.singh@univ-st-etienne.fr
}

\author{
Jean-Marie BONNIN \\ IRISA / IMT-Atlantique - Inria \\ Rennes - France \\ jean-marie.bonnin@irisa.fr
}

\begin{abstract}
In smart cities, the mobility of vehicles can be used to collect data produced by connected objects and to deliver them to several applications which are delay tolerant. The Vehicular Delay Tolerant Networks (VDTN) can be utilized for such services. This paper introduces DC4LED (Data Collection for Low Energy Devices): a hierarchical VDTN routing which takes advantage of the specific mobility patterns of the various type of vehicles. It provides a low-cost delivery service for applications that need to gather data produced from the field. The idea is to propose a simple routing scheme where cars, taxis, and buses route data hierarchically in a storecarry-forward mechanism to any of the several available Internet Point-of-Presences in the city. This paper aims to explore the possibility of implementing a very simple mechanism for very simple services, as opposed to utilizing complex VDTN mechanisms proposed in the literature. Our simulations compare the performance of DC4LED routing with two legacy VDTN routing schemes which represent the extreme ends of VDTN routing spectrum: First-contact and Epidemic routing. It show that DC4LED has much lower network overhead in comparison with the two legacy routing schemes, which is advantageous for its implementation scalability. The DC4LED also maintains comparable data delivery probability and latency to Epidemic routing.
\end{abstract}

\section{INTRODUCTION}

Smart cities today, and in the future, will have to cope with huge amounts of data coming from various low energy devices, such as wireless sensors and connectedobjects, for multiple kinds of applications. Some of these applications will be delay-tolerant such as the collection of air pollution or temperature data, smart metering, photos reporting of road degradation, etc. [1][2]. These applications will be able to tolerate data delays ranging from a few minutes to some hours. Typical ways to collect data using cellular networks and dedicated networks such as LPWAN might neither be sufficient nor efficient to handle the entire deluge of data, where the bandwidth limitation and the cost of subscription might be the limiting factors. Thus, alternative means to offload part of the delay-tolerant data will become increasingly necessary.

Simultaneously, in the landscape of vehicular technology, the capability of vehicles to communicate with each other (V2V) as well as to connected-objects in their surroundings (V2I) is becoming a norm. Vehicles will also have the capability to connect to the internet with current and future radio access networks. Furthermore, the regulatory body in Europe and US will soon make such communications capability compulsory for vehicles to support the safety-related applications [3]. This exciting new development will pave the way for vehicles to participate actively in Smart Cities ecosystems.

Figure 1 illustrates a vehicle-based data collection architecture for smart cities, where stakeholders deploy wireless sensors and associated application servers. Each sensor knows to which application server it has to send the information it produces. The data collection service could be provided for free by the city to develop its IoT ecosystem. Data for delay-tolerant application generated by a set of wireless sensors across the city need to be delivered to its associated central server. The system utilizes Vehicular Delay-Tolerant Networks (VDTN) with its store-carry-forward mechanism to gather and deliver data to one of the wireless Internet Point-of-Presence (PoP) available in the city. Note that the goal of routing for the anticipated data collection is to reach one of the available fixed positioned $\mathrm{PoP}$ in the city as fast as possible. It is not needed to reach a specific destination, which could have even been a mobile destination. Thus, the routing algorithm could be far less complex than the existing protocols proposed in the literature. This paper aims to show that a simple algorithm could be efficient enough for most of the usages.

This work proposes and evaluates DC4LED (Data Collection for Low Energy Devices): a hierarchical VDTN routing, which sensibly takes into account several common features of mobility in smart cities (e.g., buses, taxis, and cars), and hierarchically defines their role in forwarding the data. We then evaluate the algorithm using a set of simulation scenarios to acquire its baseline performances for an application-agnostic vehicle-based smart city-wide data collection service.

\section{RELATED WORKS}

In the literature, there are a lot of legacy routing schemes; a survey of VDTN routing approaches is 


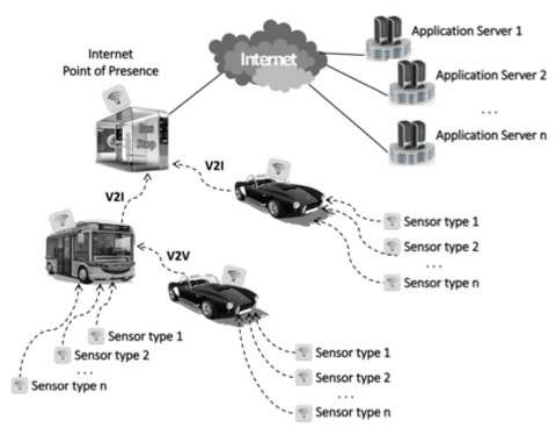

Fig. 1. Vehicle-Based Data Collection Architecture for Smart Cities

provided in [1]. However, most of them are too complex to be implemented in cars, not because of a lack of computational capabilities, but because such mechanisms need to be standardized in international bodies. The standardization environment of ITS (Intelligent Transportation System) is very complex, and if the mechanism to be standardized is too complex, it will take a while to reach a consensus, even if realizable. The data collection mechanism needs to be as simple and as generic as possible to cope with multiple service scenarios and serve a broad range of applications which have few wellidentified characteristics. Moreover, it will be possible to collect data from objects with minimum modification to their behaviors.

Previous work in [4] described the use of DTN-based architecture implementing multiple technologies to collect various kinds of data in smart cities. Numerous types of mobile nodes are considered such as cars, bicycles, and drones. They applied and evaluated Epidemic Routing strategy for message forwarding. Their real-world evaluations highlighted the problems of the flooding approach which waste a lot of network resources and causes a lot of packet drops due to buffer size limits. Their results showed that Epidemic routing reached a better delivery ratio and a lower latency compared to their proposed strategies, while on the network overhead their approach was about $75 \%$ more efficient. In our work, we focus more on the utilization of vehicles to efficiently forward data without too much complexity. Through simulations, our work also allows a performance assessment over a larger city-wide area, as well as showing the effect of increases in vehicular mobility.

\section{VDTN RouTING}

In this section, we elaborate on the need for having a simple VDTN routing scheme. Then we propose DC4LED (Data Collection for Low Energy Devices): a hierarchical VDTN routing scheme.

\section{A. Routing Ecosystem}

We focus on data collection in cities using VDTN routing. Such vehicular routing ecosystem is very heterogeneous. The data collection chain in a city starts from sensors and then the data is routed using cars, taxis, buses, etc., to an Internet PoP and then finally to Server. We argue that each of these steps in the routing chain has their complexities and constraints. For example, existing works assume routing using advanced strategies such as using multiple copies, GPS information, using an adaptive mechanism, or even social networking strategy among vehicles [5][6]. However, the feasibility and practicality of such adaptive strategies are arguably low. The adaptive mechanism may be implemented in sensors as well as servers which in turn are dedicated equipment of the service. However, as pointed out before, the standardization environment of ITS (Intelligent Transportation System) is very complex. Thus, it is difficult to assume that specialized as well as intelligent crosslayer approaches, such as multiple copies or using GPS information, will be easy to standardize in common cars. Additionally, we also discuss that the behavior and mobility pattern of each entity is different, which in turn has an impact on routing protocol design. Therefore, we review some of the entities below:

- Sensors: It has energy constraints, but can realize adaptive strategies such as forwarding multiple copies or adapting the routing according to the type of vehicle encountered by it.

- Cars: One may envision that only general and simple routing schemes will be standardized and implemented for common cars. Thus, we assume that cars will only provide simple forwarding such as single copy forwarding without necessarily using advanced information such as that of GPS. Also, we notice that cars will have mobility patterns corresponding to a very few trips per day, sometimes with very long stop times. Therefore, cars are not a very reliable entity for forwarding data.

- Taxis: As taxis are operator controlled, one can assume that it will be possible to implement some advanced routing schemes. Service provisioning business can act as motivation for taxi operators. If we consider their mobility pattern, then taxis generally have multiple trips in a day. The places visited range from random places in the city to a more specific area of interests and particular points such as taxi stands. Thus forwarding data to taxis can have some advantages such that they are more likely to visit many points in a city during the day.

- Buses: As with taxis, buses are also operator controlled and thus advanced routing can also be assumed here. Concerning the mobility, buses have a very deterministic mobility pattern, unless there is congestion in the city. Therefore, buses are a reliable DTN routing option as they visit fixed places and their trajectory is very predictable.

- PoP: It is the internet access point. One cannot assume it to be dedicated for the data collection 
service. Hence, only general Internet routing may be assumed here.

- Server: As server will already be dedicated, and for now we assume that the data collection service is only one way, from the sensor to the server.

Initialize Nodes: Server.Level $=6$; PoP.Level $=5$;

Tram.Level $=4$; Bus.Level $=3$; Taxi.Level $=2$;

Car.Level = 1; Sensor.Level = 0;

Input: Connected NeighbourNode

while (CurrentNode.Level $!=0$ ) do

if CurrentNode.Level < NeighbourNode.Level then

forward message; end

end

Algorithm 1: DC4LED Routing Forwarding Decision.

\section{B. DC4LED: A Hierarchical VDTN Routing Algorithm}

Most legacy routing approaches for VDTN such as Epidemic , Spray and Wait, MaxProp , and PRoPHET use the multiple-copy approach. However, for such schemes, the work in [2] underlined the high network overhead caused by the high number of redundant message copies. Thus, here we propose DC4LED: a hierarchical VDTN Routing scheme. For a baseline evaluation of our approach, we choose to use a singlecopy routing. Our approach sensibly takes into account common features of mobility in smart cities such as buses with fixed routes and stops as well as taxi services and cars. The fact that buses follow fixed routes makes it advantageous to deploy PoP along its path which ensures that data will be forwarded to the central server. Cars and taxis, on the other hand, can roam streets which are not passed by buses and can gather data from sensors located close to any streets in the city. The main difference between cars and taxis is in their stationary time during transit, where cars generally stop longer than taxis. Thus, we can assume that taxis are more reliable to deliver data than cars.

The Algorithm 1 describes the forwarding decisions of the simple Hierarchical Routing scheme. The idea is to statistically assign a level to the nodes in the city, instead of having complex routing decisions and metrics. This level is based on their reliability and capability to deliver the data to the Server. Note that a node does not forward the data to another node if that node's hierarchical level is inferior or equal to the current node. Thus, a bus or a taxi will not forward the data to a car, etc. There may be some missed forwarding opportunities due to this, but the idea is to keep forwarding decision as simple as possible. The performance will be extensively studied later in the paper.

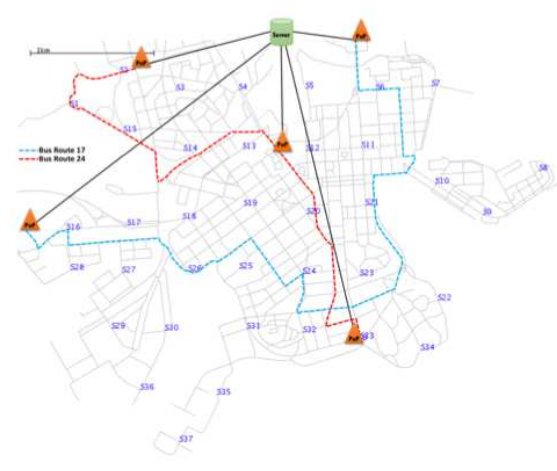

Fig. 2. Simulation Overview

\section{Simulation SETUP}

An overview of our simulations is shown in Fig. 2. It illustrates a centralized air pollution monitoring in the city of Helsinki, where 37 wireless sensors were placed almost evenly in an area of about $9 \mathrm{~km}^{2}$. Each sensor positioned approximately 500 meters to each other which gave one air quality sample data from 37 locations in the city every 5 minutes. Cars, taxis, and buses equipped with V2X capabilities then opportunistically collect data from in-range sensors to be delivered to the first PoP it encounters, which in turn relays data to a central server. We used two bus routes in the city, bus routes 17 and 24 , where a PoP is strategically placed at each end of bus routes. The last PoP is positioned at the city center where traffic usually converges, which in total makes 5 PoPs available to pick up data from cars, taxis, and buses. We evaluate the performance of the Hierarchical VDTN Routing algorithm by using the Opportunistic Networking Environment (ONE) simulator [7].

We assess the performance with a fix number of buses ( 2 for each bus route) and taxis ( 9 taxis, correspond to taxi density of 1 per $\mathrm{km}^{2}$ ), and an increasing number of cars roaming the city, from 9 to 54 with an increase of 9 each time, which correspond to car density of 1 to 6 per $\mathrm{km}^{2}$. We run each scenario ten times with different initial positioning of cars and taxis in the city, and accumulate all results to come up with convincing trends.

\section{RESUlTS AND DISCUSSION}

Fig. 3 and 4 present the comparison of our DC4LED routing with two simple legacy VDTN routing approaches which represent the two extreme ends of the DTN routing family: First-Contact (FC) which is a single-copy strategy [8] and Epidemic (EP) which is a multiple-copy flooding strategy [9]. Both can be categorized as blind forwarding approach. The only increased complexity of DC4LED as compared to both is the process of differentiating the node level to forward the messages hierarchically. The comparison are shown with increasing value of car density. It can be seen that in different scenarios, DC4LED performs better than FC 


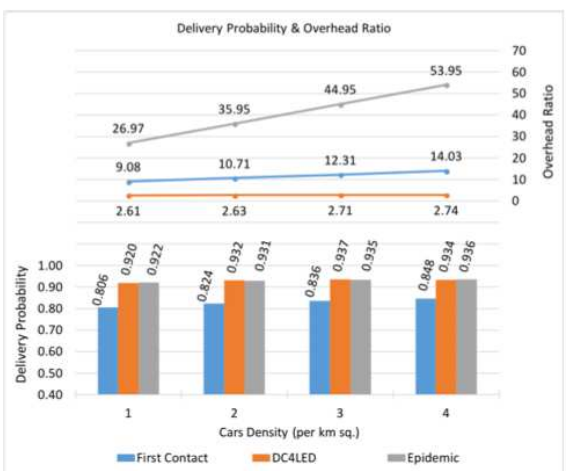

Fig. 3. Comparison of delivery probability and overhead ratio

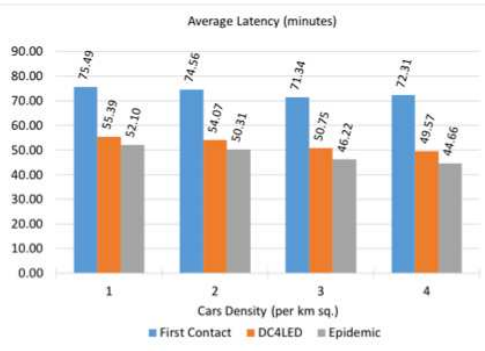

Fig. 4. Comparison of average latency

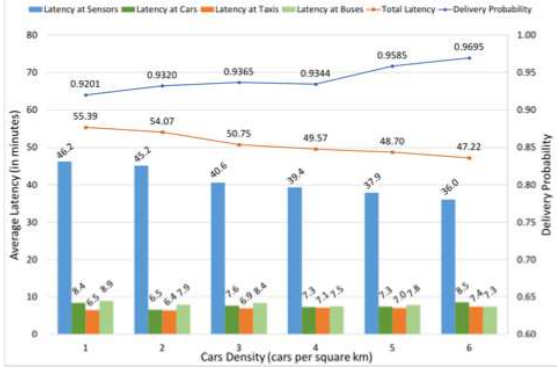

Fig. 5. Delivery probability and average latency

and almost similar to Epidemic in terms of delivery probability. By examining the average latency, DC4LED has on average 4 minutes higher latency, as compared to Epidemic. The result is expected as Epidemic uses flooding, which however has a disadvantage in that it wastes network resources. Flooding also leads to the circulation of multiple redundant copies in the network which is apparent in Fig. 3. It shows the network overhead to be highest for Epidemic and lowest for the proposed DC4LED scheme, where the overhead for the DC4LED scheme only increased slightly with the increasing number of vehicles, in contrast to the two legacy routings. This fact is advantageous for the scalability of DC4LED's implementation.

Fig. 5 summarizes two Key Performance Indicators (KPI) for the DC4LED Routing: the message delivery probability and the average latency. It contains three separate graphs, where the first part shows that a high delivery probability of 0.92 could already be reached when car density is 1 per $\mathrm{km}^{2}$. The positive trend continues when the car density increases further. It also shows the decreasing trend of messages delivery latency from about 55 minutes with a car density of 1 per $\mathrm{km}^{2}$ to about 47 minutes when car density increased to 6 per $\mathrm{km}^{2}$. Furthermore, the fact that we only include a fixed number of taxis, buses, and PoPs in the simulation points toward the high possibility to improve the KPI by increasing the number of involved taxis and buses for the data collection and by deploying more PoPs.

\section{CONCLUSION}

Simulation results for DC4LED show that the hierarchical VDTN routing scheme has a low network overhead which is advantageous for its implementation scalability. It can also achieve high data delivery probability even in a low vehicles density. We argued that the complexity of existing VDTN routing schemes is not necessary for the kind of data collection service we envisioned in smart cities. Due to our strong but realistic assumptions for the service that we have in mind, our much simpler mechanism is sufficient for the data collection task.

\section{ACKNOWLEDGMENT}

This work is supported by a Doctoral Scholarship from the Indonesian Endowment Fund for Education (LPDP) of the Indonesian Ministry of Finance, under Contract Number PRJ-43/LPDP.3/2016.

\section{REFERENCES}

[1] N. Benamar, K. D. Singh, M. Benamar, D. El Ouadghiri, and J.-M. Bonnin, "Routing protocols in Vehicular Delay Tolerant Networks: A comprehensive survey," Computer Communications, vol. 48, pp. 141-158, Jul. 2014

[2] T. Abdelkader, K. Naik, A. Nayak, N. Goel, and V. Srivastava, "A performance comparison of delay-tolerant network routing protocols," IEEE Network, vol. 30, no. 2, pp. 46-53, Mar. 2016.

[3] Z. MacHardy, A. Khan, K. Obana, and S. Iwashina, "V2x Access Technologies: Regulation, Research, and Remaining Challenges," IEEE Communications Surveys Tutorials, pp. 1-1, 2018.

[4] R. Almeida, R. Oliveira, M. Luis, C. Senna, and S. Sargento, "Forwarding Strategies for Future Mobile Smart City Networks," in 2018 IEEE 87th Vehicular Technology Conference (VTC Spring), Jun. 2018, pp. 1-7.

[5] S. H. Ahmed, H. Kang, and D. Kim, "Vehicular Delay Tolerant Network (VDTN): Routing perspectives," in 2015 12th Annual IEEE Consumer Communications and Networking Conference (CCNC), Jan. 2015, pp. 898-903.

[6] Y. Zhu, B. Xu, X. Shi, and Y. Wang, "A Survey of Social-Based Routing in Delay Tolerant Networks: Positive and Negative Social Effects," IEEE Communications Surveys Tutorials, vol. 15, no. 1, pp. 387-401, 2013.

[7] A. Keranen, J. Ott, and T. Karkkainen, "The ONE Simulator for DTN Protocol Evaluation," in Proceedings of the 2nd International Conference on Simulation Tools and Techniques, 2009, pp. 55:155:10.

[8] S. Jain, K. Fall, and R. Patra, "Routing in a Delay Tolerant Network," in Proceedings of the 2004 Conference on Applications, Technologies, Architectures, and Protocols for Computer Communications, ser. SIGCOMM '04. New York, NY, USA: ACM, 2004, pp. $145-158$.

[9] A. Vahdat and D. Becker, "Epidemic Routing for PartiallyConnected Ad Hoc Networks," Technical Report CS-200006, Duke University, Apr. 2000. 